Acknowledgements

Funding This ECHO project was supported by funding from the Northern Ireland Health and Social Care Board.

\section{Compliance with ethical standards}

Conflict of interest The authors declare that they have no conflict of interest.

\section{Reference}

1. Reeves BC, Scott LJ, Taylor J, Hogg R, Rogers CA, Wordsworth S, et al. The Effectiveness, cost-effectiveness and acceptability of Community versus Hospital Eye Service followup for patients with neovascular age-related macular degeneration with quiescent disease (ECHoES): a virtual randomised balanced incomplete block trial. Health Technol Assess. 2016;20: $1-120$.

\title{
Post-injection endophthalmitis in eyes receiving vs. not receiving topical antibiotic prophylaxis in Northern Thailand
}

\author{
Voraporn Chaikitmongkol $^{1} \cdot$ Onnisa Nanegrungsunk ${ }^{2} \cdot$ Direk Patikulsila $^{1} \cdot$ Janejit Choovuthayakorn ${ }^{1}$. \\ Nawat Watanachai ${ }^{1} \cdot$ Paradee Kunavisarut $^{1} \cdot$ Nimitr Ittipunkul $^{1} \cdot$ Neil M. Bressler $^{3}$
}

Received: 8 February 2018 / Revised: 26 April 2018 / Accepted: 7 May 2018 / Published online: 14 August 2018

(c) The Royal College of Ophthalmologists 2018

\section{Introduction}

Endophthalmitis following intravitreous anti-vascular endothelial growth factor (VEGF) injection is a rare sight-threatening condition. Several Western references suggest lower rates of post-injection endophthalmitis can be achieved without topical antibiotic prophylaxis [1-3]. However, a majority of physicians in the Asia-Pacific region still prescribe antibiotic prophylaxis [4], anecdotally stating concern that differences in environmental factors (e.g., tropical climates) or patient factors in the region might cause higher risk of endophthalmitis, comparing to the Western settings, to warrant this prophylaxis, despite little scientific rationale supporting such use. To our knowledge, little is known regarding incidence of post-injection endophthalmitis without antibiotic

Neil M. Bressler

nmboffice@jhmi.edu

1 Retina Division, Department of Ophthalmology, Faculty of Medicine, Chiang Mai University, Chiang Mai, Thailand

2 Department of Ophthalmology, Faculty of Medicine, Chiang Mai University, Chiang Mai, Thailand

3 Retina Division, Wilmer Eye Institute, Johns Hopkins University School of Medicine, Baltimore, MD, USA prophylaxis in a developing countries in Asia or elsewhere. Therefore, this study determined incidence of postinjection endophthalmitis with vs. without topical antibiotic prophylaxis at a university-based practice in Northern Thailand.

\section{Materials and methods}

IRB-approved prospective case series of patients receiving anti-VEGF injections at Chiang Mai University Hospital between May 2015 and September 2016 with follow-up anticipated for $\geq 3$ weeks after injections were recruited. Before injections, study eyes were examined by slit-lamp biomicroscopy, ensuring no intraocular inflammation. Endophthalmitis was evaluated at $4 \pm 1$ weeks after the injection, and defined as severe inflammation in both anterior chamber and vitreous cavity associated with pain, redness, or decreased vision, regardless of whether subsequent cultures were positive.

A standardized intravitreous anti-VEGF injection protocol was strictly applied, including use of sterile drape, sterile eyelid speculum, sterile glove, procedure mask, and application of povidone-iodine, twice, over eyelid and conjunctival sac, and over injection site using a povidone-iodine soaked cotton tip for $\geq 30 \mathrm{~s}$ before injection. 
Table 1 Demographics of participants and study eyes in each subgroup (receiving vs. not receiving antibiotics) and overall

\begin{tabular}{|c|c|c|c|}
\hline & \multicolumn{2}{|c|}{ Topical antibiotic prophylaxis } & \multirow{2}{*}{$\begin{array}{l}\text { Overall, } 1043 \\
\text { participants }(\%)\end{array}$} \\
\hline & $\begin{array}{l}\text { Receiving, } 381 \\
\text { participants (\%) }\end{array}$ & $\begin{array}{l}\text { Not receiving, } 662 \\
\text { participants }(\%)\end{array}$ & \\
\hline Age (mean $\pm \mathrm{SD}$, years) & $61.6 \pm 7.8$ & $60.4 \pm 7.8$ & $60.8 \pm 7.8$ \\
\hline \multicolumn{4}{|l|}{ Gender } \\
\hline Men & $193(50.7)$ & $333(50.3)$ & $526(50.4)$ \\
\hline Women & $188(49.3)$ & $329(49.7)$ & $517(49.6)$ \\
\hline \multicolumn{4}{|l|}{ Nationality } \\
\hline Thai & $377(99.0)$ & $656(99.1)$ & $1033(99.0)$ \\
\hline Hill-tribe & $4(1.0)$ & $6(0.9)$ & $10(1.0)$ \\
\hline \multicolumn{4}{|l|}{ Occupation } \\
\hline $\begin{array}{l}\text { Office workers/indoor } \\
\text { workers/retirees }\end{array}$ & $317(83.2)$ & $539(81.4)$ & $856(82.1)$ \\
\hline Outdoor workers $^{\mathrm{a}}$ & $64(16.8)$ & $123(18.6)$ & $187(17.9)$ \\
\hline \multicolumn{4}{|l|}{ Educational level } \\
\hline Primary school or lower & $219(57.5)$ & 408(61.6) & $627(60.1)$ \\
\hline $\begin{array}{l}\text { Secondary school or } \\
\text { higher }\end{array}$ & $162(42.5)$ & $254(38.4)$ & $416(39.9)$ \\
\hline \multicolumn{4}{|l|}{ Study eye } \\
\hline Unilateral & $346(90.8)$ & $565(85.3)$ & $911(87.3)$ \\
\hline \multirow[t]{2}{*}{ Bilateral } & $35(9.2)$ & $97(14.7)$ & $132(12.7)$ \\
\hline & $\begin{array}{l}\text { Receiving, } 1407 \\
\text { injections }(\%)\end{array}$ & $\begin{array}{l}\text { Not receiving, } 2856 \\
\text { injections }(\%)\end{array}$ & $\begin{array}{l}\text { Overall, } 4263 \\
\text { injections }(\%)\end{array}$ \\
\hline \multicolumn{4}{|l|}{ Indication for injection ${ }^{\mathrm{b}}$} \\
\hline DME & 445 (31.6) & $1124(39.4)$ & $1569(36.8)$ \\
\hline PCV & $206(14.6)$ & $574(20.1)$ & $780(18.3)$ \\
\hline NV AMD & $303(21.5)$ & $371(13.0)$ & $674(15.8)$ \\
\hline RVO with ME & $253(18.0)$ & $441(15.4)$ & $694(16.3)$ \\
\hline $\begin{array}{l}\text { PDR-related condition e.g., } \\
\text { VH }\end{array}$ & $138(9.8)$ & $220(7.7)$ & $358(8.4)$ \\
\hline Others & $62(4.4)$ & $126(4.4)$ & $188(4.4)$ \\
\hline \multicolumn{4}{|l|}{ Anti-VEGF agents ${ }^{c}$} \\
\hline Aflibercept & $216(15.4)$ & $758(26.5)$ & $974(22.8)$ \\
\hline Bevacizumab & $1104(78.5)$ & 2011(70.4) & $3115(73.1)$ \\
\hline Ranibizumab & $87(6.2)$ & $87(3.0)$ & $174(4.1)$ \\
\hline
\end{tabular}

${ }^{a}$ For example, agricultural workers, construction workers

${ }^{\mathrm{b}} \mathrm{DME}$ diabetic macular edema, PCV polypoidal choroidal vasculopathy, NV AMD neovascular age-related macular degeneration, RVO retinal vein occlusion, ME macular edema, PDR proliferative diabetic retinopathy, VH vitreous hemorrhage, anti-VEGF anti-vascular endothelial growth factor

${ }^{c}$ Bevacizumab was re-packaged in sterile fashion by the hospital pharmacy department into multiple doses of $1.25 \mathrm{mg} / 0.05 \mathrm{ml}$. Each dose was stored in a sterile insulin syringe, while ranibizumab $(0.5 \mathrm{mg} / 0.05 \mathrm{ml})$ and aflibercept $(2.0 \mathrm{mg} / 0.05 \mathrm{ml})$ were prepared by physicians at the time of each injection
Subsequently, participants received or did not receive topical antibiotics depending on their physicians' preferences. Three of six retina specialists (DP, PK, NI) always prescribed antibiotics $\left(\right.$ Tobramycin $^{\circledR}$ ) before or after injections for 5-7 days; the other three (JC, $\mathrm{NW}, \mathrm{VC}$ ) did not prescribe any antibiotics as part of standard care.

\section{Results}

Of 4263 injections (1043 participants) meeting eligibility criteria, 1407 (33\%) received antibiotic prophylaxis, and $2856(67 \%)$ did not receive any antibiotics. Demographics are shown in Table 1. There was one case of culture-negative post-injection endophthalmitis, which subsequently underwent vitrectomy, among eyes 
Table 2 Incidence of endophthalmitis in eyes receiving vs. not receiving antibiotic prophylaxis

\begin{tabular}{lllll}
\hline & $\begin{array}{l}\text { Topical antibiotic } \\
\text { prophylaxis }\end{array}$ & $\begin{array}{l}\text { Difference of incidence }(95 \% \\
\text { confidential interval) }\end{array}$ & P value \\
\cline { 2 - 3 } & Receiving $^{\mathrm{a}}$ & Not receiving & \\
\hline $\begin{array}{l}\text { Number of intravitreous } \\
\text { injections }\end{array}$ & 1407 & 2856 & & \\
\begin{tabular}{l} 
Incidence of endophthalmitis \\
\hline
\end{tabular} & $1(0.07 \%)$ & $-(0 \%)$ & $0.07 \%(-0.12 \%$ to $0.26 \%)$ & 0.72 \\
\hline
\end{tabular}

Tobramycin eye drops for 5-7 days before or after intravitreous injection or both before and after injection receiving antibiotics (1/1407 injections; 0.07\%). No endophthalmitis occurred among eyes not receiving antibiotics $(0 / 2856$ injections; $0 \%)$ for a difference of $0.07 \%$ ( $95 \%$ confidence interval: -0.12 to $0.26 \%$, $P=0.728$; Table 2).

\section{Discussion}

To our knowledge, this study is among the first to explore an incidence of post-injection endophthalmitis when no antibiotic prophylaxis was given in a developing-country setting. The setting, a government hospital which mainly serves lower socioeconomic patients within geographically tropical climates, may be a good representative for individuals believed by some to be at higher risk for post-injection endophthalmitis in developing countries. The results, though not a randomized clinical trial, did not reveal an increased incidence of endophthalmitis in eyes not receiving antibiotic prophylaxis, similar to Western reports [1-3]. These data support continued use of povidone-iodine for $\geq 30 \mathrm{~s}$ prior to intravitreous injection [5] but not topical antibiotics for prophylaxis against post-injection endophthalmitis.

Funding Funding of this study was provided by Research Committee, Faculty of Medicine, Chiang Mai University, and unrestricted donations to Johns Hopkins University for retina research. The sponsor had no role in the design or conduct of this research.

\section{Compliance with ethical standards}

Conflict of interest All authors have completed and submitted the ICMJE Form for Disclosure of Potential Conflicts of Interest. VC reported receiving research grant from Bayer and ThromboGenics; and receiving honoraria and travel reimbursement from Allergan and Novartis. ON reported no financial disclosure. DP reported working as a consultant, receiving honoraria and travel reimbursement from Bayer and Novartis; and receiving honoraria and travel reimbursement from Alcon. JC and NW reported receiving honoraria and travel reimbursement from Alcon, Allergan, Bayer and Novartis. PK reported receiving honoraria and travel reimbursement from Novartis. NI reported no financial disclosure. NMB reported receiving grants to employer, Johns Hopkins University; and from Bayer, Novartis, Roche (Genentech) and Samsung. No other disclosures were reported.

\section{References}

1. Bhavsar AR, Stockdale CR, Ferris FL 3rd, Brucker AJ, Bressler NM, Glassman AR. Diabetic Retinopathy Clinical Research Network. Update on risk of endophthalmitis after intravitreal drug injections and potential impact of elimination of topical antibiotics. Arch Ophthalmol. 2012;130:809-10.

2. Storey P, Dollin M, Pitcher J, Reddy S, Vojtko J, Vander J, et al. Post-Injection Endophthalmitis Study Team. The role of topical antibiotic prophylaxis to prevent endophthalmitis after intravitreal injection. Ophthalmology. 2014;121:283-9.

3. Meredith TA, McCannel CA, Barr C, Doft BH, Peskin E, Maguire MG, Comparison of Age-Related Macular Degeneration Treatments Trials Research Group. et al. Postinjection endophthalmitis in the comparison of age-related macular degeneration treatments trials (CATT). Ophthalmology. 2015;122:817-21.

4. American Society of Retinal Specialists. 2014 global trends in retina. 2014. https://www.asrs.org/content/documents/2014_globa 1_trends_comprehensivepostmtg.pdf. Accessed 20 April 2018.

5. Bhavsar AR, Glassman AR, Stockdale CR, Jampol LM. Diabetic Retinopathy Clinical Research Network. Elimination of topical antibiotics for intravitreous injections and the importance of using povidone-iodine: update from the Diabetic Retinopathy Clinical Research Network. JAMA Ophthalmol. 2016;134:1181-3. 